\title{
Markov Property and Ergodicity of the Nonlinear Filter
}

\author{
A.G. Bhatt* \\ A. Budhiraja ${ }^{\dagger}$ \\ Indian Statistical Institute, Department of Mathematics \\ New Delhi \\ University of Notre Dame \\ Notre Dame, IN 46656 \\ R. L. Karandikar* \\ Indian Statistical Institute, \\ New Delhi
}

December 11, 2000

${ }^{*}$ Research supported by Department of Science and Technology, Government of India, India

${ }^{\dagger}$ Research supported in part by the NSF grant DMI 9812857 and the University of Notre Dame Faculty Research Program 


\begin{abstract}
In this paper we first prove, under quite general conditions, that the nonlinear filter and the pair: (signal,filter) are Feller-Markov processes. The state space of the signal is allowed to be non locally compact and the observation function: $h$ can be unbounded. Our proofs in contrast to those of Kunita(1971,1991), Stettner(1989) do not depend upon the uniqueness of the solutions to the filtering equations. We then obtain conditions for existence and uniqueness of invariant measures for the nonlinear filter and the pair process. These results extend those of Kunita and Stettner, which hold for locally compact state space and bounded $h$, to our general framework. Finally we show that the recent results of Ocone-Pardoux [11] on asymptotic stability of the nonlinear filter, which use the Kunita-Stettner setup, hold for the general situation considered in this paper.
\end{abstract}

Key Words: nonlinear filtering, invariant measures, asymptotic stability, measure valued processes.

AMS Classification:60 G 35, 60 J 05, 60 H 15 


\section{Introduction}

Kunita's pioneering paper [9] showed the way for analyzing asymptotic behavior of the non-linear filter. Kunita showed for the case of compact state space valued Markov signal process that the non-linear filter itself is a Markov process. This proof was based on the fact that the filter is the unique solution to the Kushner-Stratonovich equation (or the Fujisaki- KallianpurKunita equation). Kunita also showed that if the signal is ergodic then so is the filter. These results were extended to the case of locally compact state space (with a bounded observation function $h$ ) by Kunita [10] and Stettner [12]. The proofs of these results also crucially used the uniqueness of the solution to the filtering equation.

In this article, we first show that in the signal and noise independent case (which is also the case treated in the above stated papers), when the signal is a Markov process, then so is the filter. This proof does not depend upon uniqueness of filtering equations. It just uses the Bayes formula to explicitly evaluate various conditional expectations and deduce that the filter is Markov. Using recent results in Bhatt et. al. [2], we deduce that under fairly general conditions on $h$, the filter is a Feller Markov process. We also show that under the same conditions, the pair process: (signal, filter) is a Feller-Markov process. Once this is done, we can obtain results of Kunita [10], Stettner [12] and Ocone-Pardoux [11] on ergodicity and asymptotic stability of the filter in our framework, where the state space is a complete separable metric space and the function $h$ is allowed to be unbounded and minimal conditions are imposed on it.

Let us begin with listing some common notation used in this article. For a complete separable metric space $S$, let $C(S)$ be the class of continuous functions on $S, C_{b}(S)$ be the class of bounded continuous functions on $S$, $\mathcal{B}(S)$ be the Borel $\sigma$-field on $S, \mathcal{P}(S)$ be the space of probability measures on $(S, \mathcal{B}(S))$ endowed with the weak convergence topology, $\mathcal{M}_{+}(S)$ be the class of positive finite measures on $(S, \mathcal{B}(S))$ with the weak convergence topology, $D([0, \infty), S)$ be the class of functions, which are right continuous and have left limits (r.c.l.l.), from $[0, \infty)$ into $S$ with Skorokhod topology and $C([0, \infty), S)$ be the class of continuous functions from $[0, \infty)$ into $S$ with topology of uniform convergence on compact subsets of $[0, \infty)$. 


\section{Preliminaries}

Consider the non-linear filtering model

$$
Y_{t}=\int_{0}^{t} h\left(X_{u}\right) d u+W_{t}
$$

where $\left(X_{t}\right)$ is the signal process, taking values in a complete separable metric space $E, h: E \rightarrow \mathbb{R}^{d}$ is a continuous mapping, $\left(W_{t}\right)$ is a $\mathbb{R}^{d}$-valued standard Wiener process, assumed to be independent of $\left(X_{t}\right)$. $\left(Y_{t}\right)$ is the observation process. $\left(X_{t}\right),\left(W_{t}\right)$ are defined on a probability space $(\Omega, \mathcal{F}, P)$.

The object of interest in filtering theory is the conditional distribution $\pi_{t}$ of $X_{t}$ given the observations up until time $t$, i.e. given $\sigma\left(Y_{u} ; u \leq t\right)$.

We are going to assume that the signal process $\left(X_{t}\right)$ is a Markov process with transition probability function $p(s, x, t, B)$ : for $0 \leq s<t, x \in E$, $B \in \mathcal{B}(E)$,

$$
P\left(X_{t} \in B \mid \sigma\left(X_{u}: u \leq s\right)\right)=p\left(s, X_{s}, t, B\right) \text { a.s. }
$$

The initial distribution of $\left(X_{t}\right)$ will be denoted by $\gamma$, i.e.

$$
\gamma=P \circ\left(X_{0}\right)^{-1} \text {. }
$$

Let $\xi_{t}(\cdot)$ be the coordinate process on $\mathcal{D} \doteq D([0, \infty), E)$, i.e. $\xi_{t}(\theta) \doteq \theta(t)$ for $\theta \in \mathcal{D}$. We assume that for all $(s, x) \in[0, \infty) \times E$ there exists a probability measure $P_{s, x}$ on $\mathcal{D}$ such that for $0 \leq s<t<\infty$, and $U \in \mathcal{B}(E)$,

$$
P_{s, x}\left(\xi_{t} \in U \mid \sigma\left(\xi_{u}: u \leq s\right)\right)=p\left(s, \xi_{s}, t, U\right) \text { a.s. } P_{s, x}
$$

and

$$
P_{s, x}\left(\xi_{u}=x, 0 \leq u \leq s\right)=1 .
$$

Further, we assume that the mapping

$$
(s, x) \longrightarrow P_{s, x} \text { is continuous. }
$$

As usual, for $\nu \in \mathcal{P}(E)$, and $B \in \mathcal{B}(\mathcal{D})$, let

$$
P_{s, \nu}(B) \doteq \int P_{s, x}(B) \nu(d x), \quad B \in \mathcal{B}(\mathcal{D}) .
$$


Note that

$$
P \circ(X)^{-1}=P_{0, \gamma} .
$$

The Markov property implies that $\left(T_{t}\right)$ defined below is a semigroup:

$$
\left(T_{t} f\right)(s, x) \doteq \int f(s+t, y) p(s, x, s+t, d y), \quad(s, x) \in[0, \infty) \times E
$$

for $f \in C_{b}([0, \infty) \times E), \quad 0 \leq t<\infty$. The assumption (2.6) implies that $\left(T_{t}\right)$ is a Feller semigroup i.e.

$$
T_{t}\left(C_{b}([0, \infty) \times E)\right) \subset C_{b}([0, \infty) \times E) .
$$

It is well known (and easy to verify) that (2.9) implies that $\left(X_{t}\right)$ is continuous in probability. As a consequence, the filter $\left(\pi_{t}\right)$ admits a continuous version (see [3]) .

In order to study the Markov properties of $\left(\pi_{t}\right)$ we first present the following preliminary result.

\section{Path wise integration formula.}

We cite the following result from [8]. As we shall see in the next section, this formulation of stochastic integral is very useful when dealing with a family of measures on a measurable space.

The result is Theorem 3 in [8]. The mapping $\mathcal{I}$ is explicitly constructed in that paper.

Theorem 3.1 There exists a measurable mapping $\mathcal{I}: D([0, \infty), \mathbb{R}) \times$ $D([0, \infty), \mathbb{R}) \rightarrow D([0, \infty), \mathbb{R})$ with the property that if $\left(U_{t}\right)$ is a semimartingale (assumed to have r.c.l.l. paths) on a probability space $\left(\Omega^{\prime}, \mathcal{F}^{\prime}, P^{\prime}\right)$ with respect to a filtration $\left(\mathcal{F}_{t}^{\prime}\right)$ and if $\left(V_{t}\right)$ is an r.c.l.l. $\left(\mathcal{F}_{t}^{\prime}\right)$ - adapted process, then

$$
Z_{t}\left(w^{\prime}\right):=\mathcal{I}\left(V \cdot\left(w^{\prime}\right), U \cdot\left(w^{\prime}\right)\right)(t), \quad w^{\prime} \in \Omega^{\prime}
$$


is a version of the stochastic integral $\int_{0}^{t} V_{-} d U$, i.e.

$$
Z_{t}=\int_{0}^{t} V_{s-} d U_{s} \quad \forall t \text { a.s. } P^{\prime} .
$$

\section{Markov properties of the filter:}

In this section we will study the Markov properties of the filter. The first main result is Theorem 4.5 in which we show that $\left\{\pi_{t}, \sigma\left\{Y_{u}: u \leq t\right\}\right\}$ is a Markov process on $(\Omega, \mathcal{F}, P)$. The second central result of this section is Theorem 4.9 in which we show that if $\bar{\pi}_{t}$ is a sub-optimal filter defined via an incorrect initialization of the filtering process (to be made precise later in the section) then $\left\{\left(\bar{\pi}_{t}, X_{t}\right), \mathcal{F}_{t}\right\}$ is a Markov process on $(\Omega, \mathcal{F}, P)$ with state space $\mathcal{P}(E) \times E$, where $\mathcal{F}_{t} \doteq \sigma\left\{\left(X_{s}, Y_{s}\right): s \leq t\right\}$. In particular, this result implies that $\left\{\left(\pi_{t}, X_{t}\right), \mathcal{F}_{t}\right\}$ is a Markov process on $(\Omega, \mathcal{F}, P)$.

Let $\beta_{t}(\cdot)$ be the coordinate process on $\mathcal{C} \doteq C\left([0, \infty), \mathbb{R}^{d}\right)$, i.e. $\beta_{t}(\eta) \doteq \eta(t)$ for $\eta \in \mathcal{C}$. Let $Q$ be the standard Wiener measure on $(\mathcal{C}, \mathcal{B}(\mathcal{C}))$. Let

$$
(\hat{\Omega}, \hat{\mathcal{F}}) \doteq(\mathcal{D}, \mathcal{B}(\mathcal{D})) \otimes(\mathcal{C}, \mathcal{B}(\mathcal{C}))
$$

and for $0 \leq s<\infty, \quad \nu \in P(E)$,

$$
R_{s, \nu} \doteq P_{s, \nu} \otimes Q .
$$

Let

$$
Z_{t}(\theta, \eta) \doteq \sum_{j=1}^{d} \mathcal{I}\left(h^{j}(\xi(\theta)), \beta^{j}(\eta)\right)(t),
$$

where $h(x) \equiv\left(h^{1}(x), \cdots, h^{d}(x)\right)$ and $\beta_{t}(\eta) \equiv\left(\beta_{t}^{1}(\eta), \cdots, \beta_{t}^{d}(\eta)\right)$ for $x \in E$ and $\eta \in \mathcal{C}$.

Since $\left(\beta_{t}\right)$, considered as a process on $(\hat{\Omega}, \hat{\mathcal{F}})$, is a Wiener process under $R_{s, \nu}$, it follows from Theorem 3.1 that

$$
Z_{t}-Z_{s}=\sum_{j=1}^{d} \int_{s}^{t} h^{j}\left(\xi_{u}\right) d \beta_{u}^{j} \quad \text { a.s. } R_{s, \nu}
$$


for every $0 \leq s<t<\infty$ and $\nu \in \mathcal{P}(E)$. The main thing to note is that we have been able to construct a common version of the stochastic integral appearing in (4.2) for the family of probability measures $\left\{R_{s, \nu}\right\}$.

For $0 \leq s<t<\infty$, let

$$
q_{s t}(\theta, \eta) \doteq \exp \left(Z_{t}(\theta, \eta)-Z_{s}(\theta, \eta)-\frac{1}{2} \sum_{j=1}^{d} \int_{s}^{t}\left(h^{j}\left(\xi_{u}(\theta)\right)^{2} d u\right) .\right.
$$

It is well known (and easy to verify) that $\left\{q_{s t}: t \geq s\right\}$ is a $\left\{R_{s, \nu}\right\}$ martingale for every $\nu \in \mathcal{P}(E)$.

Let us note that for $0 \leq s<t$,

$$
q_{0 t}(\theta, \eta)=q_{0 s}(\theta, \eta) q_{s t}(\theta, \eta) \quad \forall(\theta, \eta) \in \hat{\Omega} .
$$

For $0 \leq s<t<\infty, \eta \in \mathcal{C}$ and $\nu \in \mathcal{M}_{+}(E)$, let $\Gamma_{s t}(\nu, \cdot)(\eta) \in \mathcal{M}_{+}(E)$ and $\Lambda_{s t}(\nu, \cdot)(\eta) \in \mathcal{P}(E)$ be defined as follows. For $B \in \mathcal{B}(E)$

$$
\Gamma_{s t}(\nu, B)(\eta) \doteq \int_{E} \int_{\mathcal{D}} 1_{B}\left(\xi_{t}(\theta)\right) q_{s t}(\theta, \eta) d P_{s, x}(\theta) d \nu(x)
$$

and

$$
\Lambda_{s t}(\nu, B)(\eta) \doteq \Gamma_{s t}(\nu, B)(\eta) / \Gamma_{s t}(\nu, E)(\eta) .
$$

The measure $\Gamma_{s t}(\nu, \cdot)(\eta)$ will also be denoted by $\Gamma_{s t}(\nu)(\eta)$ and likewise, $\Lambda_{s t}(\nu, \cdot)(\eta)$ will be denoted by $\Lambda_{s t}(\nu)(\eta)$. Since $\mathbb{E}_{R_{s, \nu}}\left[q_{s t}\right]=1$, it follows that

$$
\mathbb{E}_{Q}\left[\Gamma_{s t}(\nu, E)\right]=\nu(E)
$$

Note that for $\nu \in \mathcal{M}_{+}(E)$, if $\hat{\nu}$ is defined by

$$
\hat{\nu}(B) \doteq \frac{\nu(B)}{\nu(E)},
$$

then

$$
\Gamma_{s t}(\nu, B)(\eta)=\nu(E) \Gamma_{s t}(\hat{\nu}, B)(\eta)
$$

and

$$
\Lambda_{s t}(\nu, B)(\eta)=\Lambda_{s t}(\hat{\nu}, B)(\eta) .
$$

As a consequence of the Kallianpur-Striebel formula (See [1], [2], [6], [7]) it follows that

$$
\pi_{t}(\omega)(B) \doteq \Lambda_{0 t}(\gamma, B)(Y .(\omega))
$$

is a version of the filter $\mathbb{E}_{P}\left[1_{B}\left(X_{t}\right) \mid \sigma\left(Y_{u}: u \leq t\right)\right]$. Furthermore, a.s. $P, \pi_{t}$ has continuous paths. (See [3]). Here is a technical result needed later. 
Theorem 4.1 Fix $0 \leq s<t, \nu \in \mathcal{P}(E)$. Let $\mathcal{F}^{*}$ be the completion of $\hat{\mathcal{F}}$ under $R_{0, \nu}$. Let $\mathcal{N}$ be the class of $R_{0, \nu}$ null sets in $\mathcal{F}^{*}$. Considering $\xi, \beta$ as processes defined on $\left(\hat{\Omega}, \mathcal{F}^{*}, R_{0, \nu}\right)$, let us define the following sub- $\sigma$ fields of $\mathcal{F}^{*}:$

$$
\begin{aligned}
\mathcal{G}_{s}^{t} & =\sigma\left(\sigma\left(\xi_{u}: s \leq u \leq t\right) \cup \mathcal{N}\right) \\
\mathcal{E}_{0} & =\sigma\left(\sigma\left(\beta_{u}: u \geq 0\right) \cup \mathcal{N}\right) \\
\mathcal{E}_{1} & =\sigma\left(\mathcal{G}_{0}^{s} \cup \mathcal{E}_{0}\right) \\
\mathcal{E}_{2} & =\sigma\left(\mathcal{G}_{s}^{\infty} \cup \mathcal{E}_{0}\right) .
\end{aligned}
$$

Let $g$ be a $\mathcal{E}_{2}$ - measurable, $R_{0, \nu}$-integrable random variable. Then

$$
\mathbb{E}_{R_{0, \nu}}\left[g \mid \mathcal{E}_{1}\right]=g_{1}, \text { a.s. }
$$

where

$$
g_{1}(\theta, \eta)=\int_{\mathcal{D}} g\left(\theta_{1}, \eta\right) d P_{s, \xi_{s}(\theta)}\left(\theta_{1}\right)
$$

Remark 4.1 In (4.11) above, when $t=\infty$, the right hand side is to be interpreted as $\sigma\left(\sigma\left(\xi_{u}: s \leq u<\infty\right) \cup \mathcal{N}\right)$.

Proof : When $g$ is $\mathcal{G}_{s}^{\infty}$ - measurable bounded random variable, the result follows from the Markov property of the family $\left\{P_{s, x}\right\}$ and independence of $\beta$ and $\xi$ under $R_{0, \nu}$. When $g$ is of the form

$$
g=\sum_{j=1}^{k} g_{j} f_{j}
$$

where $g_{j}$ are $\mathcal{G}_{s}^{\infty}$-measurable bounded functions and $f_{j}$ are $\mathcal{E}_{0}$ measurable, bounded functions the result follows from the preceding observation. Since the class of functions as in (4.13) forms an algebra that generates the $\sigma$-field $\mathcal{E}_{2}$, the result follows.

The next result connects $\left\{\Gamma_{s t}\right\}$ with each other and is a key step in the proof of the Markov property. 
Theorem 4.2 Fix $0 \leq s<t<\infty, \nu \in \mathcal{P}(E)$. Then

$$
\Gamma_{0 t}(\nu, B)(\eta)=\Gamma_{s t}\left(\Gamma_{0 s}(\nu)(\eta), B\right)(\eta), \quad \forall B \in \mathcal{B}(E), \quad \eta-\text { a.s. }[Q]
$$

and

$$
\Lambda_{0 t}(\nu, B)(\eta)=\Lambda_{s t}\left(\Lambda_{0 s}(\nu)(\eta), B\right)(\eta), \quad \forall B \in \mathcal{B}(E), \quad \eta-\text { a.s. }[Q]
$$

Proof : Fix $A \in \mathcal{B}(\mathcal{C}), B \in \mathcal{B}(E)$. Let

$$
G(A, B) \doteq \int_{\mathcal{C}} \Gamma_{0 t}(\nu, B)(\eta) 1_{A}(\eta) d Q(\eta) .
$$

Using Fubini's theorem, definition of $\Gamma_{s t}$ and the relation (4.4), it follows that

$$
G(A, B)=\int_{E} \int_{\hat{\Omega}} 1_{A}(\eta) q_{0 s}(\theta, \eta) g(\theta, \eta) d R_{0, x}(\theta, \eta) d \nu(x) .
$$

where

$$
g(\theta, \eta)=1_{B}\left(\xi_{t}(\theta)\right) q_{s t}(\theta, \eta) .
$$

Let $\mathcal{E}_{1}, \mathcal{E}_{2}$ be as in Theorem 4.1. Note that $1_{A}(\eta) q_{0 s}(\theta, \eta)$ is $\mathcal{E}_{1}$ - measurable and $g$ is $\mathcal{E}_{2}$-measurable. It follows from Theorem 4.1 that

$$
\mathbb{E}_{R_{0, x}}\left[g \mid \mathcal{E}_{1}\right](\theta, \eta)=f\left(\xi_{s}(\theta), \eta\right) \quad \text { a.s. }(\theta, \eta)\left[R_{0, x}\right]
$$

where

$$
\begin{aligned}
f(x, \eta) & \doteq \int_{\mathcal{D}} g\left(\theta_{1}, \eta\right) d P_{s, x}\left(\theta_{1}\right) \\
& =\int_{\mathcal{D}} 1_{B}\left(\xi_{t}\left(\theta_{1}\right)\right) q_{s t}\left(\theta_{1}, \eta\right) d P_{s, x}\left(\theta_{1}\right)
\end{aligned}
$$

Before proceeding, let us note that for $\nu \in P(E)$

$$
\int_{E} f(y, \eta) d \nu(y)=\Gamma_{s t}(\nu, B)(\eta) .
$$

Using (4.16), (4.17) and the fact that $1_{A}(\eta) q_{0 s}(\theta, \eta)$ is $\mathcal{E}_{1^{-}}$measurable, it follows that

$$
G(A, B)=\int_{E} \int_{\hat{\Omega}} 1_{A}(\eta) q_{0 s}(\theta, \eta) f\left(\xi_{s}(\theta), \eta\right) d R_{0, x}(\theta, \eta) d \nu(x) .
$$


Now applying the definition of $\Gamma_{0 s}$, we have that

$$
G(A, B)=\int_{\mathcal{C}} 1_{A}(\eta)\left[\int_{E} f(y, \eta) \Gamma_{0 s}(\nu, d y)(\eta)\right] d Q(\eta)
$$

and as a consequence we have on using (4.18) that

$$
G(A, B)=\int_{\mathcal{C}} 1_{A}(\eta) \Gamma_{s t}\left(\Gamma_{0 s}(\nu)(\eta), B\right)(\eta) d Q(\eta) .
$$

Since (4.19) holds for all $A \in \mathcal{B}(\mathcal{C})$, and the sigma field $\mathcal{B}(E)$ is countably generated, the relation (4.14) follows. The identity (4.15) is a consequence of (4.14) and (4.8)-(4.9).

We are now in a position to prove that $\left\{\Gamma_{0 t}: t \geq 0\right\},\left\{\Lambda_{0 t}: t \geq 0\right\}$ are Markov processes on $(\mathcal{C}, \mathcal{B}(\mathcal{C}), Q)$ with state spaces $\mathcal{M}_{+}(E)$ and $\mathcal{P}(E)$ respectively. Let $\tilde{\mathcal{F}}$ be the $Q$ - completion of $\mathcal{B}(\mathcal{C})$ and $\tilde{\mathcal{N}}$ be the class of $Q$ null sets in $\tilde{\mathcal{F}}$. For $0 \leq s \leq t \leq \infty$, let $\mathcal{A}_{s}^{t}$ be the sub $\sigma$-fields of $\tilde{\mathcal{F}}$ defined by

$$
\mathcal{A}_{s}^{t}=\sigma\left(\sigma\left(\beta_{u}-\beta_{s}: s \leq u \leq t\right) \cup \tilde{\mathcal{N}}\right) .
$$

Here and in what follows, we will consider $\beta$ and $\xi$ as processes on $\hat{\Omega}$. It is easy to see that

$$
\Gamma_{0 t}(\nu), \Lambda_{0 t}(\nu) \text { are } \mathcal{A}_{0}^{t} \text { measurable }
$$

and for $s<t$

$$
\Gamma_{s t}(\nu), \Lambda_{s t}(\nu) \text { are } \mathcal{A}_{s}^{\infty} \text { measurable. }
$$

These observations lead us to the following theorem.

Theorem 4.3 Let $\nu \in \mathcal{P}(E)$. Then $\left(\Gamma_{0 t}(\nu), \mathcal{A}_{0}^{t}\right)$ and $\left(\Lambda_{0 t}(\nu), \mathcal{A}_{0}^{t}\right)$ are Markov processes on $(\mathcal{C}, \tilde{\mathcal{F}}, Q)$. Furthermore, for fixed $0 \leq s<t<\infty$, and real valued Borel measurable functions $\psi$ and $\varphi$ on $\mathcal{M}_{+}(E)$ and $\mathcal{P}(E)$, respectively, which satisfy:

$$
\mathbb{E}_{Q}\left[\left|\psi\left(\Gamma_{u t}(\lambda)\right)\right|\right]<\infty
$$

and

$$
\mathbb{E}_{Q}\left[\left|\varphi\left(\Lambda_{u t}(\lambda)\right)\right|\right]<\infty
$$


for all $0 \leq u \leq t$ and $\lambda \in \mathcal{M}_{+}(E)$ we have that

$$
\begin{aligned}
& \mathbb{E}_{Q}\left[\psi\left(\Gamma_{0 t}(\nu)\right) \mid \mathcal{A}_{0}^{s}\right]=\psi_{1}\left(\Gamma_{0 s}(\nu)\right) \\
& \mathbb{E}_{Q}\left[\varphi\left(\Lambda_{0 t}(\nu)\right) \mid \mathcal{A}_{0}^{s}\right]=\varphi_{1}\left(\Lambda_{0 s}(\nu)\right)
\end{aligned}
$$

where

$$
\psi_{1}(\lambda) \doteq \mathbb{E}_{Q}\left[\psi\left(\Gamma_{s t}(\lambda)\right)\right], \quad \lambda \in \mathcal{M}_{+}(E)
$$

and

$$
\varphi_{1}(\nu) \doteq \mathbb{E}_{Q}\left[\varphi\left(\Lambda_{s t}(\nu)\right)\right], \quad \nu \in \mathcal{P}(E) .
$$

Proof : We will only prove the result for the case where $\varphi$ and $\psi$ are bounded. The general case follows by the usual approximation arguments (using the observation (4.7)).

We have observed that

$$
\psi\left(\Gamma_{0 t}(\nu)\right)=\psi\left(\Gamma_{s t}\left(\Gamma_{0 s}(\nu)\right)\right),
$$

$\Gamma_{0 s}(\nu)$ is $\mathcal{A}_{0}^{s}$ measurable and $\Gamma_{s t}(\lambda)$ is $\mathcal{A}_{s}^{\infty}$ measurable (for all $\lambda \in \mathcal{M}_{+}(E)$ ). Also, $\mathcal{A}_{0}^{s}$ and $\mathcal{A}_{s}^{\infty}$ are independent under $Q$. This implies (4.23) with $\psi_{1}$ defined by (4.25).

For $\varphi \in C_{b}(\mathcal{P}(E))$ define $\psi \in C_{b}\left(\mathcal{M}_{+}(E)\right)$ by

$$
\psi(\lambda) \doteq \varphi\left(\frac{1}{\lambda(E)} \lambda\right) .
$$

Then, for $\nu \in \mathcal{P}(E)$

$$
\varphi\left(\Lambda_{0 t}(\nu)\right)=\psi\left(\Gamma_{0 t}(\nu)\right)
$$

As a result

$$
\mathbb{E}_{Q}\left[\varphi\left(\Lambda_{0 t}(\nu)\right) \mid \mathcal{A}_{0}^{s}\right]=\psi_{1}\left(\Gamma_{0 s}(\nu)\right) .
$$

where for $\lambda \in \mathcal{M}_{+}(E)$

$$
\begin{aligned}
\psi_{1}(\lambda) & =\mathbb{E}_{Q}\left[\psi\left(\Gamma_{s t}(\lambda)\right)\right] \\
& =\mathbb{E}_{Q}\left[\varphi\left(\Lambda_{s t}(\lambda)\right)\right] .
\end{aligned}
$$


Given $\lambda \in \mathcal{M}_{+}(E)$, define $\hat{\lambda} \in \mathcal{P}(E)$ by

$$
\hat{\lambda}(B) \doteq \frac{1}{\lambda(E)} \lambda(B), \quad B \in \mathcal{B}(E) .
$$

Then from (4.9) and definition of $\phi_{1}$ (see (4.26)), we have that,

$$
\begin{aligned}
\phi_{1}(\hat{\lambda}) & =\mathbb{E}_{Q}\left[\varphi\left(\Lambda_{s t}(\hat{\lambda})\right)\right] \\
& =\mathbb{E}_{Q}\left[\varphi\left(\Lambda_{s t}(\lambda)\right)\right] \\
& =\psi_{1}(\lambda) .
\end{aligned}
$$

As a consequence,

$$
\psi_{1}\left(\Gamma_{0 s}(\nu)\right)=\phi_{1}\left(\Lambda_{0 s}(\nu)\right) .
$$

This in view of (4.27) proves (4.24).

As noted earlier, on $\left(\hat{\Omega}, \hat{\mathcal{F}}, R_{s, \nu}\right), \nu \in \mathcal{P}(E)$,

$$
\left(q_{s t}\right)_{t \geq s} \text { is a martingale w.r.t. } \sigma\left(\xi_{u}, \beta_{u}: u \leq t\right)
$$

and hence that

$$
\rho_{s t}= \begin{cases}\Gamma_{s t}(\nu, E), & t \geq s \\ 1 & t \leq s\end{cases}
$$

is a martingale on $(\mathcal{C}, \mathcal{B}(\mathcal{C}), Q)$ w.r.t. $\left(\mathcal{A}_{0}^{t}\right)$. Let $Q_{s, \nu} \in P(\mathcal{C})$ be defined by

$$
\frac{d Q_{s, \nu}}{d Q}=\rho_{s t} \text { on } \mathcal{A}_{0}^{t} .
$$

From Girsanov's theorem, it follows that $Q_{0, \gamma}$ is the law of the observation process $Y$, i.e. $P o Y^{-1}=Q_{0, \gamma}($ See $[6])$.

We are going to prove that $\left\{\Gamma_{0 t}(\nu)\right\},\left\{\Lambda_{0 t}(\nu)\right\}$ are Markov processes on $\left(\mathcal{C}, \mathcal{B}(\mathcal{C}), Q_{0, \nu}\right)$ as well.

Theorem 4.4 Let $\nu \in \mathcal{P}(E)$. Then $\left(\Gamma_{0 t}(\nu), \mathcal{A}_{0}^{t}\right)$ and $\left(\Lambda_{0 t}(\nu), \mathcal{A}_{0}^{t}\right)$ are Markov processes on $\left(\mathcal{C}, \tilde{\mathcal{F}}, Q_{0, \nu}\right)$. Furthermore , for $f \in C_{b}\left(\mathcal{M}_{+}(E)\right), g \in$ $C_{b}(\mathcal{P}(E))$,

$$
\mathbb{E}_{Q_{0, \nu}}\left[f\left(\Gamma_{0 t}(\nu)\right) \mid \mathcal{A}_{0}^{s}\right]=f_{1}\left(\Gamma_{0 s}(\nu)\right)
$$


and

$$
\mathbb{E}_{Q_{0, \nu}}\left[g\left(\Lambda_{0 t}(\nu)\right) \mid \mathcal{A}_{0}^{s}\right]=g_{1}\left(\Lambda_{0 s}(\nu)\right) .
$$

where $f_{1}, g_{1}$ are defined as follows. For $\lambda \in \mathcal{M}_{+}(E), \nu \in \mathcal{P}(E)$

$$
f_{1}(\lambda) \doteq \mathbb{E}_{Q_{s, \hat{\lambda}}}\left[f\left(\Gamma_{s t}(\lambda)\right)\right]
$$

where $\hat{\lambda}(A) \doteq \frac{1}{\lambda(E)} \lambda(A)$ and

$$
g_{1}(\nu) \doteq \mathbb{E}_{Q_{s, \nu}}\left[g\left(\Lambda_{s t}(\nu)\right)\right]
$$

Proof : We will first prove (4.30). Fix $0 \leq s<t<\infty, \nu \in \mathcal{P}(E)$ and $A \in \mathcal{A}_{0}^{s}$. Then

$$
\begin{aligned}
\int_{A} f\left(\Gamma_{0 t}(\nu)\right) d Q_{0, \nu} & =\int_{A} f\left(\Gamma_{0 t}(\nu)\right) \Gamma_{0 t}(\nu, E) d Q \\
& =\int_{A} \psi\left(\Gamma_{0 t}(\nu)\right) d Q \\
& =\int_{A} \psi_{1}\left(\Gamma_{0 s}(\nu)\right) d Q
\end{aligned}
$$

where $\psi(\lambda) \doteq f(\lambda) \lambda(E)$ and $\psi_{1}$ is given by (4.25). Thus defining $f_{1}(\lambda)=$ $\frac{\psi_{1}(\lambda)}{\lambda(E)}$, it follows that

$$
\begin{aligned}
\int_{A} f\left(\Gamma_{0 t}(\nu)\right) d Q_{0, \nu} & =\int_{A} f_{1}\left(\Gamma_{0 s}(\nu)\right) \Gamma_{0 s}(\nu, E) d Q \\
& =\int_{A} f_{1}\left(\Gamma_{0 s}(\nu)\right) d Q_{0, \nu}
\end{aligned}
$$

and hence (4.30) holds. Further note that

$$
\begin{aligned}
f_{1}(\lambda) & =\frac{1}{\lambda(E)} \psi_{1}(\lambda) \\
& =\frac{1}{\lambda(E)} \int_{\mathcal{C}} f\left(\Gamma_{s t}(\lambda)\right) \Gamma_{s t}(\lambda, E) d Q \\
& =\int_{\mathcal{C}} f\left(\Gamma_{s t}(\lambda)\right) \Gamma_{s t}(\hat{\lambda}, E) d Q \\
& =\mathbb{E}_{Q_{s, \hat{\lambda}}}\left[f\left(\Gamma_{s t}(\lambda)\right)\right] .
\end{aligned}
$$


Thus, $f_{1}$ is given by (4.32).

Now we prove (4.31). Let $g \in C_{b}(P(E))$ be as in the statement of the theorem. Let $f \in C_{b}\left(\mathcal{M}_{+}(E)\right)$ be defined as

$$
f(\lambda) \doteq g\left(\frac{1}{\lambda(E)} \lambda\right)
$$

so that $f\left(\Gamma_{0 t}(\nu)\right)=g\left(\Lambda_{0 t}(\nu)\right)$. Thus,

$$
\mathbb{E}_{Q_{0, \nu}}\left[g\left(\Lambda_{0 t}(\nu)\right) \mid \mathcal{A}_{0}^{s}\right]=f_{1}\left(\Gamma_{0 s}(\nu)\right) .
$$

where $f_{1}$ is given by (4.32). Note that,

$$
\begin{aligned}
f_{1}(\lambda) & =\mathbb{E}_{Q_{s, \hat{\lambda}}}\left[f\left(\Gamma_{s t}(\lambda)\right)\right] \\
& =\mathbb{E}_{Q_{s, \hat{\lambda}}}\left[g\left(\frac{1}{\Gamma_{s t}(\lambda, E)} \Gamma_{s t}(\lambda)\right)\right] \\
& =\mathbb{E}_{Q_{s, \hat{\lambda}}}\left[g\left(\Lambda_{s t}(\lambda)\right)\right] \\
& =\mathbb{E}_{Q_{s, \hat{\lambda}}}\left[g\left(\Lambda_{s t}(\hat{\lambda})\right)\right] \\
& =g_{1}(\hat{\lambda}),
\end{aligned}
$$

where $g_{1}$ is defined by (4.33). Thus we have in particular that

$$
\mathbb{E}_{Q_{0, \nu}}\left[g\left(\Lambda_{0 t}(\nu)\right) \mid \mathcal{A}_{0}^{s}\right]=f_{1}\left(\Gamma_{0 s}(\nu)\right)=g_{1}\left(\Lambda_{0 s}(\nu)\right) .
$$

An immediate consequence of the above result is that for bounded measurable function $G$ on $\mathcal{P}(E)$,

$$
\left(\mathcal{T}_{s t} G\right)(\nu) \doteq \mathbb{E}_{Q_{s, \nu}}\left[G\left(\Lambda_{s t}(\nu)\right)\right]
$$

defines a two parameter semigroup, i.e for $0 \leq s<t<u$ :

$$
\mathcal{T}_{s t} \circ \mathcal{T}_{t u}=\mathcal{T}_{s u} .
$$

Moreover, as noted earlier, $\pi_{t}(\omega)=\Lambda_{0 t}(\gamma)(Y .(\omega))$ is a version of the filter $\mathbb{E}_{P}\left[X_{t} \in . \mid Y_{u}: u \leq t\right]$, while $Q_{0, \gamma}$ is the law of the observation process $Y$. Thus we have the following result.

Theorem $4.5\left\{\pi_{t}\right\}$ is a $\mathcal{P}(E)$ - valued Markov process on $(\Omega, \mathcal{F}, P)$ with associated two parameter semigroup $\left\{\mathcal{T}_{\text {st }}\right\}$. 
Further, if the signal process $\left(X_{t}\right)$ is a time homogeneous Markov process then so is $\left\{\pi_{t}\right\}$ with

$$
\mathcal{T}_{s t}=\mathcal{T}_{0 u} ; \quad u=t-s .
$$

Proof : The first part follows easily from the preceding theorem. For the second part, let us note that the law of $\left\{\left(\xi_{s+u}, \beta_{s+u}-\beta_{s}\right): u \geq 0\right\}$ under $R_{s, \gamma}$ is $R_{0, \gamma}$. As a consequence, law of $\Gamma_{s t}(\gamma)$ under $R_{s, \gamma}$ is same as the law of $\Gamma_{0,(t-s)}(\gamma)$ under $R_{0, \gamma}$.

This yields the required result.

Let $\nu \in \mathcal{P}(E)$. Define the process:

$$
\bar{\pi}_{t}(\omega) \doteq \Lambda_{0 t}(\nu)(Y .(\omega)) .
$$

Then $\left(\bar{\pi}_{t}\right)$ considered as a process on $(\Omega, \mathcal{F}, P)$ represents a sub-optimal filtering process which has been initiated at the incorrect initial law $\nu$ rather than the actual initial law $\gamma$. In the final part of this section we study the Markov properties of the process $\left(\bar{\pi}_{t}, X_{t}\right)$. The importance of Markov property and ergodicity of the pair process in the study of asymptotic robustness questions in nonlinear filtering has been pointed out in $[4,5]$. Define the stochastic process $\left(\Psi_{t}^{\nu}\right)$ on $(\hat{\Omega}, \hat{\mathcal{F}})$ with values in $\mathcal{P}(E) \times E$ as follows.

$$
\Psi_{t}^{\nu}(\theta, \eta) \doteq\left(\Lambda_{0 t}(\nu)(\eta), \xi_{t}(\theta)\right) .
$$

Let $\mathcal{G}_{s}^{t}$ be as in Theorem 4.1, where $\mathcal{N}$ is the class of all $R_{0, \gamma}$ null sets. $\mathcal{G}_{s}^{t}$ are sub-sigma fields of the completion $\mathcal{F}^{*}$ of $\mathcal{F}$ under the measure $R_{s, \gamma}$. We now define the following sub-sigma fields of $\mathcal{F}^{*}$ : for $0 \leq s \leq t \leq \infty$

$$
\begin{gathered}
\mathcal{H}_{s}^{t} \doteq \sigma\left(\sigma\left\{\beta_{u}-\beta_{s}: s \leq u \leq t\right\} \cup \mathcal{N}\right), \\
\mathcal{K}_{s}^{t} \doteq \sigma\left(\mathcal{G}_{s}^{t} \cup \mathcal{H}_{s}^{t}\right) .
\end{gathered}
$$

(See Remark 4.1 above for the case $t=\infty$.) The following theorem is essentially a consequence of Theorem 4.3.

Theorem 4.6 Let $\gamma, \nu \in \mathcal{P}(E)$. Then $\left(\Psi_{t}^{\nu}, \mathcal{K}_{0}^{t}\right)$ is a Markov process on $\left(\hat{\Omega}, \hat{F}, R_{0, \gamma}\right)$. Furthermore, for $0 \leq s<t<\infty$ and a bounded measurable function $f$ on $\mathcal{P}(E) \times E$ we have

$$
\mathbb{E}_{R_{0, \gamma}}\left[f\left(\Lambda_{0 t}(\nu), \xi_{t}\right) q_{0 t} \mid \mathcal{K}_{0}^{s}\right]=f_{1}\left(\Lambda_{0 s}(\nu), \xi_{s}\right) q_{0 s} \text { a.s. }
$$


where $f_{1}: \mathcal{P}(E) \times E \rightarrow \mathbb{R}$ is defined as follows: for $(\lambda, x) \in \mathcal{P}(E) \times E$

$$
f_{1}(\lambda, x) \doteq \mathbb{E}_{R_{s, x}}\left[f\left(\Lambda_{s t}(\lambda), \xi_{t}\right) q_{s t}\right]
$$

Proof: The Markov property of $\left(\Psi_{t}^{\nu}, \mathcal{K}_{0}^{t}\right)$ is a direct consequence of Theorem 4.3 on noting that under $R_{0, \gamma}$ the sigma fields $\mathcal{G}_{0}^{s}, \mathcal{H}_{0}^{s}$ are independent and $\left(\Lambda_{0 t}(\nu), \mathcal{H}_{0}^{t}, R_{0, \gamma}\right)$ and $\left(\xi_{t}, \mathcal{G}_{0}^{t}, R_{0, \gamma}\right)$ are Markov processes. We now consider the second part of the theorem. Since $q_{0 t}=q_{0 s} q_{s t}$ and $q_{s t}$ is $\mathcal{K}_{s}^{\infty}$ measurable, it suffices to prove that for a $\mathcal{K}_{s}^{\infty}$ measurable random variable $Z$ with $\mathbb{E}_{R_{0, \gamma}}[|Z|]<\infty$

$$
\mathbb{E}_{R_{0, \gamma}}\left[f\left(\Lambda_{0 t}(\nu), \xi_{t}\right) Z \mid \mathcal{K}_{0}^{s}\right]=f_{2}\left(\Lambda_{0 s}(\nu), \xi_{s}\right) \text { a.s. },
$$

where $f_{2}: \mathcal{P}(E) \times E \rightarrow \mathbb{R}$ is defined as follows: for $(\lambda, x) \in \mathcal{P}(E) \times E$

$$
f_{2}(\lambda, x) \doteq \mathbb{E}_{R_{s, x}}\left[f\left(\Lambda_{s t}(\lambda), \xi_{t}\right) Z\right] .
$$

Using the usual approximation arguments, the proof of (4.39)-(4.40) can be reduced to the case when $f(\lambda, x)=g(\lambda) h(x)$ for bounded measurable functions $g, h$ and $Z=U V$, with $U$ being bounded $\mathcal{G}_{s}^{\infty}$ measurable and $V$ being bounded $\mathcal{H}_{s}^{\infty}$ measurable.

Note that $\mathcal{H}_{0}^{\infty}=\sigma\left(\cup_{t} \mathcal{H}_{0}^{t}\right)$ and $\mathcal{G}_{0}^{\infty}=\sigma\left(\cup_{t} \mathcal{G}_{0}^{t}\right)$ are independent under $R_{0, \gamma}$. Also, $g\left(\Lambda_{0 t}(\nu)\right) V$ is $\mathcal{H}_{0}^{\infty}$ measurable, $\mathcal{H}_{s}^{\infty} \subseteq \mathcal{H}_{0}^{\infty}, h\left(\xi_{t}\right) U$ is $\mathcal{G}_{0}^{\infty}$ measurable and $\mathcal{G}_{s}^{\infty} \subseteq \mathcal{G}_{0}^{\infty}$. Further, $\mathcal{K}_{0}^{s}=\sigma\left(\mathcal{G}_{0}^{s} \cup \mathcal{H}_{0}^{s}\right)$. These observations imply that

$$
\mathbb{E}_{R_{0, \gamma}}\left[g\left(\Lambda_{0 t}(\nu)\right) V h\left(\xi_{t}\right) U \mid \mathcal{K}_{0}^{s}\right]=\mathbb{E}_{R_{0, \gamma}}\left[g\left(\Lambda_{0 t}(\nu)\right) V \mid \mathcal{H}_{0}^{s}\right] \mathbb{E}_{R_{0, \gamma}}\left[h\left(\xi_{t}\right) U \mid \mathcal{G}_{0}^{s}\right] .
$$

In turn, using $\Lambda_{0 t}(\nu)=\Lambda_{s t}\left(\Lambda_{0 s}(\nu)\right)$ and the independence of $\mathcal{H}_{0}^{s}$ and $\mathcal{H}_{s}^{\infty}$, it follows that

$$
\mathbb{E}_{R_{0, \gamma}}\left[g\left(\Lambda_{0 t}(\nu)\right) V \mid \mathcal{H}_{0}^{s}\right]=g_{2}\left(\Lambda_{0 s}(\nu)\right)
$$

with

$$
\begin{aligned}
g_{2}(\lambda) & =\mathbb{E}_{R_{s, x}}\left[g\left(\Lambda_{s t}(\lambda)\right) V\right] \\
& =\int_{\mathcal{C}} g\left(\Lambda_{s t}(\lambda)\right) V d Q
\end{aligned}
$$

where the second display above follows on recalling that $R_{s, x}=P_{s, x} \otimes Q$. Using the Markov property of $\left(\xi_{t}\right)$ it follows that

$$
\mathbb{E}_{R_{0, \gamma}}\left[h\left(\xi_{t}\right) U \mid \mathcal{G}_{0}^{s}\right]=h_{2}\left(\xi_{s}\right)
$$


with

$$
h_{2}(x)=\mathbb{E}_{R_{s, x}}\left[h\left(\xi_{t}\right) U\right] .
$$

The equations (4.41)-(4.45) imply that (4.39) holds with $f_{2}(\lambda, x)=$ $g_{2}(\lambda) h_{2}(x)$. Using independence of $g\left(\Lambda_{s t}(\lambda)\right) V$ and $h\left(\xi_{t}\right) U$, it follows that (4.40) also holds. As noted at the beginning of the proof, this in turn implies the second part of the theorem, namely (4.37)-(4.38)

Now for fixed $\nu \in \mathcal{P}(E)$ and $s \geq 0$ define $\hat{R}_{s, \nu}$ on $(\hat{\Omega}, \hat{\mathcal{F}})$ as follows:

$$
\frac{d \hat{R}_{s, \nu}}{d R_{s, \nu}}(\theta, \eta) \doteq q_{s t}(\theta, \eta) \quad \text { on } \mathcal{K}_{0}^{t}, t \geq s .
$$

Recall the definition (4.29) of $Q_{s, \nu}$ and that $R_{s, \nu} \doteq P_{s, \nu} \otimes Q$. It now follows from (4.5) that

$$
\hat{R}_{s, \nu}(\mathcal{D} \times B)=Q_{s, \nu}(B) \quad B \in \mathcal{B}(\mathcal{C}) .
$$

The following result is the key step in the proof of the Markov property of $\left(\left(X_{t}, \bar{\pi}_{t}\right), \mathcal{F}_{t}\right)$ on $(\Omega, \mathcal{F}, P)$.

Theorem 4.7 Let $\nu \in \mathcal{P}(E)$. Then $\left(\Psi_{t}^{\nu}, \mathcal{K}_{0}^{t}\right)$ is a Markov process on $\left(\hat{\Omega}, \hat{\mathcal{F}}, \hat{R}_{0, \gamma}\right)$.

Proof: $\quad$ Fix $0 \leq s<t<\infty$. Let $f: \mathcal{P}(E) \times E \rightarrow \mathbb{R}$ be a bounded measurable function and let $A \in \mathcal{K}_{0}^{s}$. Then using (4.37) it follows that

$$
\begin{aligned}
\int_{A} f\left(\Lambda_{0 t}(\nu), \xi_{t}\right) d \hat{R}_{0, \gamma} & =\int_{A} f\left(\Lambda_{0 t}(\nu), \xi_{t}\right) q_{0 t} d R_{0, \gamma} \\
& =\int_{A} f_{1}\left(\Lambda_{0 s}(\nu), \xi_{s}\right) q_{0 s} d R_{0, \gamma} .
\end{aligned}
$$

Hence

$$
\mathbb{E}_{\hat{R}_{0, \gamma}}\left[f\left(\Lambda_{0 t}(\nu), \xi_{t}\right) \mid \mathcal{K}_{0}^{s}\right]=f_{1}\left(\Lambda_{0 s}(\nu), \xi_{s}\right)
$$

where $f_{1}$ is given by (4.38).

Remark 4.2 The relation defining $f_{1}$ can be recast as

$$
f_{1}(\lambda, x) \doteq \mathbb{E}_{\hat{R}_{s, x}}\left[f\left(\Lambda_{s t}(\lambda), \xi_{t}\right)\right] .
$$


Now we come to the Markov property of the filter and signal.

Theorem 4.8 Fix $\nu \in \mathcal{P}(E)$. Let $\bar{\pi}_{t}$ be as in (4.34). Then $\left(\left(\bar{\pi}_{t}, X_{t}\right), \mathcal{F}_{t}\right)$ is a $\mathcal{P}(E) \times E$ valued Markov process on $(\Omega, \mathcal{F}, P)$ with associated two parameter semigroup $\left\{\mathcal{S}_{s, t}\right\}_{0 \leq s<t<\infty}$ defined as follows. For a real bounded measurable map $f$ on $\mathcal{P}(E) \times E$,

$$
\left(\mathcal{S}_{s t} f\right)(\lambda, x) \doteq \mathbb{E}_{\hat{R}_{s, x}}\left[f\left(\Lambda_{s t}(\lambda), \xi_{t}\right)\right]
$$

for $(\lambda, x) \in \mathcal{P}(E) \times E$. Furthermore if $\left(X_{t}\right)$ is time homogeneous then so is $\left(\bar{\pi}_{t}, X_{t}\right)$, i.e. $\mathcal{S}_{s t}=\mathcal{S}_{0, t-s}$.

Proof: The Markov property follows on applying Theorem 4.7 and observing that the law of $\left\{\left(X_{t}, \bar{\pi}_{t}, Y_{t}\right): t \geq 0\right\}$ under $P$ is same as the law of $\left\{\left(\xi_{t}, \Lambda_{0 t}(\nu), \beta_{t}\right): t \geq 0\right\}$ under $\hat{R}_{0, \gamma}$. The identities (4.49)-(4.50) imply the assertion made about the semigroup $\mathcal{S}_{s t}$.

\section{Time homogeneous signal: Feller properties of the filter}

We will now examine the case of time homogeneous signal. In this case, we write

$$
\begin{gathered}
P_{\nu}=P_{0, \nu}, R_{\nu}=R_{0, \nu}, Q_{\nu}=Q_{0, \nu}, \hat{R}_{\nu}=\hat{R}_{0 \nu}, \\
\Gamma_{t}=\Gamma_{0 t}, \Lambda_{t}=\Lambda_{0 t}, \mathcal{T}_{t}=\mathcal{T}_{0 t}, \mathcal{S}_{t}=\mathcal{S}_{0 t} .
\end{gathered}
$$

We will now show that under a suitable condition, $\left\{\mathcal{T}_{t}\right\}$ and $\left\{\mathcal{S}_{t}\right\}$ are Feller semigroups. The key step in the proof of the Feller property is a result on robustness of the filter from [2]. It is shown there that under a suitable condition on $h, \nu_{n}$ converging to $\nu$ implies that

$$
Q_{\nu_{n}} \circ\left(\Lambda\left(\nu_{n}\right)\right)^{-1} \rightarrow Q_{\nu} \circ(\Lambda(\nu))^{-1} .
$$

This would immediately give the Feller property of $\mathcal{T}_{t}$. The following is essentially proved in [2]. We include here an outline of the proof as the exact 
result stated below is not given in [2]. This would enable us to conclude that $\mathcal{S}_{t}$ is a Feller Semigroup.

Theorem 5.1 Let $h$ be continuous map from $E$ to $\mathbb{R}^{d}$. Let $\left\{\nu_{n}\right\} \subset \mathcal{P}(E)$ be a sequence converging to $\nu$ weakly. Then the following hold.

(a) For all $t \geq 0, \Lambda_{t}\left(\nu_{n}\right) \rightarrow \Lambda_{t}(\nu)$ in $Q$ probability.

(b) For all $t>0, \Gamma_{t}\left(\nu_{n}, E\right) \rightarrow \Gamma_{t}(\nu, E)$ in $L^{1}(Q)$.

Proof: For (a) note that continuity of $h$ and continuity of the mapping $x \mapsto P_{x}$ implies that

$$
\lim _{N \rightarrow \infty}\left[\sup _{x \in K} P_{x}\left(\int_{0}^{T}\left\|h\left(\xi_{t}\right)\right\|^{2} 1_{\left\{\left\|h\left(\xi_{t}\right)\right\| \geq N\right\}} d t>\varepsilon\right)\right]=0
$$

for all $\varepsilon>0, T<\infty$ and for all compact subsets $K \subset E$. The result now follows from Theorem 3.2 in [2].

Since $\nu_{n} \rightarrow \nu$ weakly as $n \rightarrow \infty$, in view of our assumption on $\left\{P_{x}\right\}$ we have that $P_{\nu_{n}} \rightarrow P_{\nu}$ weakly as $n \rightarrow \infty$. Now let $\left\{\tilde{X}_{t}^{n}\right\}$ and $\left\{\tilde{X}_{t}\right\}$ be processes with values in $\mathcal{D}$ defined on some probability space $(\bar{\Omega}, \overline{\mathcal{F}}, \bar{P})$ such that $\mathcal{L}\left(\tilde{X}^{n}\right)=P_{\nu_{n}}, \mathcal{L}(\tilde{X}$. $)=P_{\nu}$ and $\tilde{X}^{n} \rightarrow \tilde{X}$. a.s. $\bar{P}$. Define :

$$
\left(\Omega_{0}, \mathcal{F}_{0}, R_{0}\right) \doteq(\bar{\Omega} \times \mathcal{C}, \overline{\mathcal{F}} \otimes \mathcal{B}(\mathcal{C}), \bar{P} \otimes Q)
$$

and the processes $Z^{n}, Z$. on this space as

$$
\begin{gathered}
Z_{t}^{n}(\bar{\omega}, \eta) \doteq q_{0 t}\left(\tilde{X}^{n}(\bar{\omega}), \eta\right), \\
Z_{t}(\bar{\omega}, \eta) \doteq q_{0 t}(\tilde{X}(\bar{\omega}), \eta) .
\end{gathered}
$$

Then note that

$$
\Gamma_{t}\left(\nu_{n}, B\right)(\eta)=\int_{\bar{\Omega}} 1_{B}\left(\tilde{X}^{n}(\bar{\omega})\right) Z_{t}^{n}(\bar{\omega}, \eta) d \bar{P}
$$

and

$$
\Gamma_{t}(\nu, B)(\eta)=\int_{\bar{\Omega}} 1_{B}(\tilde{X}(\bar{\omega})) Z_{t}(\bar{\omega}, \eta) d \bar{P}
$$


It is shown in [2] that $Z_{t}^{n} \rightarrow Z_{t}$ in $L^{1}\left(R_{0}\right)$ and this implies part (b).

As a consequence of the above theorem we have the following results.

Theorem 5.2 Let $h$ be continuous map from $E$ to $\mathbb{R}^{d}$. Then $\left(\mathcal{T}_{t}\right)$ is a Feller semigroup.

Proof : Let $\left\{\nu_{n}\right\}$ be a sequence in $\mathcal{P}(E)$. Suppose that $\nu_{n}$ converges weakly to $\nu$. Let $G$ be a bounded and continuous real function on $\mathcal{P}(E)$. We need to show that

$$
\left(\mathcal{T}_{t} G\right)\left(\nu_{n}\right) \rightarrow\left(\mathcal{T}_{t} G\right)(\nu)
$$

This follows from Theorem 5.1 upon observing that

$$
\begin{aligned}
\left(\mathcal{T}_{t} G\right)\left(\nu_{n}\right) & =\mathbb{E}_{Q_{\nu_{n}}}\left(G\left(\Lambda_{t}\left(\nu_{n}\right)\right)\right) \\
& =\mathbb{E}_{Q}\left(G\left(\Lambda_{t}\left(\nu_{n}\right)\right) \Gamma_{t}\left(\nu_{n}, E\right)\right)
\end{aligned}
$$

and similarly,

$$
\left(\mathcal{T}_{t} G\right)(\nu)=\mathbb{E}_{Q}\left(G\left(\Lambda_{t}(\nu)\right) \Gamma_{t}(\nu, E)\right)
$$

In the following theorem we prove the Feller property of $\left(\bar{\pi}_{t}, X_{t}\right)$.

Theorem 5.3 Let $h$ be continuous map from $E$ to $\mathbb{R}^{d}$. Then $\left\{\mathcal{S}_{t}\right\}$ is a Feller semigroup.

Proof: Let $F$ be a real bounded and continuous function on $\mathcal{P}(E) \times E$. Let $\left\{\left(\nu_{n}, x_{n}\right)\right\}$ be a sequence in $\mathcal{P}(E) \times E$ such that $\left(\nu_{n}, x_{n}\right) \rightarrow(\nu, x)$. We need to show that

$$
\left(\mathcal{S}_{t} F\right)\left(\nu_{n}, x_{n}\right) \rightarrow\left(\mathcal{S}_{t} F\right)(\nu, x) .
$$

Now observe that

$$
\begin{aligned}
\left(\mathcal{S}_{t} F\right)\left(\nu_{n}, x_{n}\right) & =\mathbb{E}_{\hat{R}_{x_{n}}}\left(F\left(\Lambda_{t}\left(\nu_{n}\right), \xi_{t}\right)\right) \\
& =\mathbb{E}_{R_{x_{n}}}\left(F\left(\Lambda_{t}\left(\nu_{n}\right), \xi_{t}\right) q_{0 t}\right) \\
& =\mathbb{E}_{R_{0}}\left(F\left(\Lambda_{t}\left(\nu_{n}\right), \tilde{X}_{t}^{n}\right) Z_{t}^{n}\right)
\end{aligned}
$$


where $\tilde{X}^{n}, Z^{n}, R_{0}$ are as in the proof of Theorem 5.1 with $\nu_{n}$ replaced by $\delta_{x_{n}}$. Now the result follows once more by an application of Theorem 5.1 and recalling that $\tilde{X}^{n} \rightarrow \tilde{X}$. a.s. as $n \rightarrow \infty$.

In the final result of this section we study the connection between the invariant measures for the semigroups $\mathcal{T}_{t}$ and $\mathcal{S}_{t}$. For a real measurable function $\varphi$ on a Polish space $S$ and a measure $\nu$ on $(S, \mathcal{B}(S))$ we will write $\int_{S} \varphi(x) d \nu(x)$ as $\nu(\varphi)$ when the former makes sense.

Proposition 5.4 Let $\bar{M}$ be a $\left(\mathcal{S}_{t}\right)$ invariant probability measure. Define the probability measures $\mu$ on $(E, \mathcal{B}(E))$ and $M$ on $(\mathcal{P}(E), \mathcal{B}(\mathcal{P}(E)))$ as follows.

$$
\mu(B) \doteq \bar{M}(\mathcal{P}(E) \times B) ; \quad B \in \mathcal{B}(E),
$$

and

$$
M(C) \doteq \bar{M}(C \times E) ; \quad C \in \mathcal{B}(\mathcal{P}(E)) .
$$

Then $\mu$ is a $\left(T_{t}\right)$ invariant measure. Furthermore, if for all real bounded measurable functions $f$ on $E$ and $F$ on $\mathcal{P}(E)$ :

$$
\int_{\mathcal{P}(E) \times E} F(\nu) f(x) \bar{M}(d \nu, d x)=\int_{\mathcal{P}(E)} \nu(f) F(\nu) M(d \nu),
$$

then $M$ is a $\left(\mathcal{T}_{t}\right)$ invariant measure.

Proof: Let $f$ be a bounded measurable function on $E$. Define $\tilde{f}$ : $\mathcal{P}(E) \times E \rightarrow \mathbb{R}$ as $\tilde{f}(\nu, x) \doteq f(x)$. Then

$$
\begin{aligned}
\int_{E}\left(T_{t} f\right)(x) \mu(d x) & =\int_{\mathcal{P}(E) \times E}\left(T_{t} f\right)(x) \bar{M}(d \nu, d x) \\
& =\int_{\mathcal{P}(E) \times E} \mathbb{E}_{\hat{R}_{x}}\left(\tilde{f}\left(\Lambda_{0 t}(\nu), \xi_{t}\right)\right) \bar{M}(d \nu, d x) \\
& =\int_{\mathcal{P}(E) \times E}\left(S_{t} \tilde{f}\right)(\nu, x) \bar{M}(d \nu, d x) \\
& =\int_{\mathcal{P}(E) \times E} \tilde{f}(\nu, x) \bar{M}(d \nu, d x) \\
& =\int_{E} f(x) \mu(d x) .
\end{aligned}
$$


This proves that $\mu$ is $\left(T_{t}\right)$ invariant.

Next let $G$ be a real bounded measurable function on $\mathcal{P}(E)$ and suppose that (5.5) holds. Then for all real bounded measurable functions $\Phi$ on $\mathcal{P}(E) \times E$,

$$
\int_{\mathcal{P}(E) \times E} \Phi(\nu, x) \bar{M}(d \nu, d x)=\int_{\mathcal{P}(E)}\left(\int_{E} \Phi(\nu, x) \nu(d x)\right) M(d \nu) .
$$

Define $\tilde{G}: \mathcal{P}(E) \times E \rightarrow \mathbb{R}$ as $\tilde{G}(\nu, x) \doteq G(\nu)$. Then, recalling (4.47)

$$
\begin{aligned}
\int_{\mathcal{P}(E)}\left(\mathcal{T}_{t} G\right)(\nu) M(d \nu) & =\int_{\mathcal{P}(E)} \mathbb{E}_{Q_{\nu}}\left(G\left(\Lambda_{t}(\nu)\right)\right) M(d \nu) \\
& =\int_{\mathcal{P}(E)} \mathbb{E}_{\hat{R}_{\nu}}\left(G\left(\Lambda_{t}(\nu)\right)\right) M(d \nu) \\
& =\int_{\mathcal{P}(E)}\left(\int_{E} \mathbb{E}_{\hat{R}_{x}}\left(G\left(\Lambda_{t}(\nu, \cdot)\right)\right) \nu(d x)\right) M(d \nu) \\
& =\int_{\mathcal{P}(E)}\left(\int_{E}\left(\mathcal{S}_{t} \tilde{G}\right)(\nu, x) \nu(d x)\right) M(d \nu) \\
& =\int_{\mathcal{P}(E) \times E}\left(\mathcal{S}_{t} \tilde{G}\right)(\nu, x) \bar{M}(d \nu, d x) \\
& =\int_{\mathcal{P}(E) \times E} \tilde{G}(\nu, x) \bar{M}(d \nu, d x) \\
& =\int_{\mathcal{P}(E)} G(\nu) M(d \nu) .
\end{aligned}
$$

This proves that $M$ is $\left(\mathcal{T}_{t}\right)$ invariant.

\section{Ergodic properties of the filter.}

In this section we will use the notations of Section 5. We will also assume throughout the rest of the paper that $h$ is a continuous function. Thus, $\left\{\mathcal{T}_{t}\right\}$ and $\left\{\mathcal{S}_{t}\right\}$ are Feller semigroups. We will obtain conditions for uniqueness of invariant measures corresponding to these semigroups. These questions have been studied in great detail for compact ([9]) and locally compact $([12,10,11])$ state spaces. The proofs of the results in the above papers rely on the uniqueness of the solution to the FKK or Kushner-Stratonovich 
equation, in fact even the proof of the Feller-Markov property of the filtering process crucially uses the uniqueness of the solution to the above mentioned equation. Nevertheless, using the methods and results of Sections 4 and 5 many statements and theorems in the above papers carry over to our general setup with almost identical proofs. We will now present the main ergodicity results for the filtering model considered in this paper. In order to avoid tedious repetition, we will provide proofs only when they differ from the proofs of the analogous statements in $([9,12,10,11])$.

Let $C_{c}(E)$ be the class of all convex functions in $C_{b}(E)$. Following Stettner ([12]), denote for $\nu \in \mathcal{P}(E)$ and $A \in \mathcal{B}(\mathcal{P}(E))$,

$$
m_{t}^{\nu}(A) \doteq\left(\mathcal{T}_{t} \mathcal{I}_{A}\right)(\nu)=E_{Q_{\nu}}\left(\mathcal{I}_{A}\left(\Lambda_{t}(\nu, \cdot)\right)\right)
$$

and

$$
M_{t}^{\nu}(A) \doteq \int_{E}\left(\mathcal{T}_{t} \mathcal{I}_{A}\right)\left(\delta_{x}\right) \nu(d x),
$$

where $\mathcal{I}_{A}$ is the indicator function of the set $A$. We will now give alternative representations for $m_{t}^{\nu}$ and $M_{t}^{\nu}$ as the laws of certain filtering processes.

We begin with the following setup. Let $\mu$ be a $\left(T_{t}\right)$ invariant measure. $\mathcal{D}_{\mathbb{R}} \equiv D((-\infty, \infty) ; E)$ will denote the space of r.c.l.l. functions from $(-\infty, \infty)$ into $E$ with Skorokhod topology and $\mathcal{C}_{\mathbb{R}} \equiv C\left((-\infty, \infty) ; \mathbb{R}^{d}\right)$ will denote the space of continuous functions from $(-\infty, \infty)$ into $\mathbb{R}^{d}$ with topology of uniform convergence on compact subsets of $(-\infty, \infty)$. Let the coordinate processes on $\mathcal{D}_{\mathbb{R}}$ and $\mathcal{C}_{\mathbb{R}}$ be denoted once more by $\left(\xi_{t}(\cdot)\right)$ and $\left(\beta_{t}(\cdot)\right)$ respectively. Let $P_{\mu}^{(1)}$ be the unique measure on $\left(\mathcal{D}_{\mathbb{R}}, \mathcal{B}\left(\mathcal{D}_{\mathbb{R}}\right)\right)$ which satisfies for $E_{1}, \cdots, E_{n} \in \mathcal{B}(\mathbb{R})$ and $-\infty<t_{1}<t_{2} \cdots<t_{n}<\infty$,

$$
\begin{aligned}
P_{\mu}^{(1)}\left(\xi_{t_{1}}\right. & \left.\in E_{1}, \cdots, \xi_{t_{n}} \in E_{n}\right) \\
& =\int_{E_{1} \times \cdots \times E_{n}} \mu\left(d x_{1}\right) p\left(t_{1}, x_{1}, t_{2}, d x_{2}\right) \cdots p\left(t_{n-1}, x_{n-1}, t_{n}, d x_{n}\right) .
\end{aligned}
$$

Now let $Q^{(1)}$ be a probability measure on $\left(C_{\mathbb{R}}, \mathcal{B}\left(C_{\mathbb{R}}\right)\right)$, such that for $-\infty<$ $t_{0}<t_{1} \cdots<t_{n}<\infty$,

$$
\left(\frac{1}{\sqrt{t_{1}-t_{0}}}\left(\beta_{t_{1}}-\beta_{t_{0}}\right), \cdots, \frac{1}{\sqrt{t_{n}-t_{n-1}}}\left(\beta_{t_{n}}-\beta_{t_{n-1}}\right)\right)
$$

are independent $N\left(0, I_{d \times d}\right)$. 
Now let $\Omega^{1} \doteq \mathcal{D}_{\mathbb{R}} \times C_{\mathbb{R}}$. Let $R_{\mu}^{(1)}=P_{\mu}^{(1)} \otimes Q^{(1)}$. In this section, we will consider the coordinate processes $\left(\xi_{t}\right),\left(\beta_{t}\right)$ to be defined on the product space $\left(\Omega^{1}, \mathcal{B}\left(\Omega^{1}\right), R_{\mu}^{(1)}\right)$. Define the observation process:

$$
\alpha_{t}-\alpha_{s} \doteq \int_{s}^{t} h\left(\xi_{u}\right) d u+\beta_{t}-\beta_{s} .
$$

and the observation sigma fields

$$
\mathcal{Z}_{s}^{t} \doteq \sigma\left(\alpha_{v}-\alpha_{u} ; s \leq u \leq v \leq t\right)
$$

where $-\infty \leq s<t \leq \infty$. Further, for $s, t$ such that $-\infty \leq s<t \leq \infty$, let $\mathcal{G}_{s}^{t}, \mathcal{H}_{s}^{t}$ be defined respectively by (4.11) and (4.35). The cases $s=-\infty$ and $t=\infty$ are treated as in Remark 4.1. Here, these are sub-sigma fields of $\mathcal{B}\left(\Omega^{1}\right)$. Further, let $\mathcal{G}_{-\infty}^{-\infty}$ be defined by

$$
\mathcal{G}_{-\infty}^{-\infty}=\cap_{-\infty<t<\infty} \mathcal{G}_{-\infty}^{t} .
$$

Now define for $-\infty<s<t<\infty$,

$$
\bar{\pi}_{s, t}^{(0)} \doteq \Lambda_{t-s}(\mu)\left(\alpha^{s}\right),
$$

where $\alpha^{s}: \Omega^{1} \rightarrow C\left([0, \infty) ; \mathbb{R}^{d}\right)$ is defined as $\alpha_{u}^{s}(\omega) \doteq \alpha_{s+u}(\omega)-\alpha_{s}(\omega)$. Also define

$$
\bar{\pi}_{s, t}^{(1)} \doteq \Lambda_{t-s}\left(\delta_{\xi_{s}}\right)\left(\alpha^{s}\right) .
$$

Observe that for bounded and continuous function $f$ on $E$

$$
\bar{\pi}_{s, t}^{(0)}(f)=\mathbb{E}_{R_{\mu}^{(1)}}\left[f\left(\xi_{t}\right) \mid \mathcal{Z}_{s}^{t}\right]
$$

and

$$
\bar{\pi}_{s, t}^{(1)}(f)=\mathbb{E}_{R_{\mu}^{(1)}}\left[f\left(\xi_{t}\right) \mid \mathcal{Z}_{s}^{t} \vee \sigma\left(\xi_{s}\right)\right] .
$$

(for two sigma fields $\mathcal{L}_{1}$ and $\mathcal{L}_{2}, \mathcal{L}_{1} \vee \mathcal{L}_{2} \doteq \sigma\left(\mathcal{L} 1 \cup \mathcal{L}_{2}\right)$.) Also note that for real bounded measurable function $F$ on $\mathcal{P}(E)$

$$
\begin{aligned}
\mathbb{E}_{R_{\mu}^{(1)}}\left[F\left(\bar{\pi}_{s, t}^{(1)}\right)\right] & =\mathbb{E}_{R_{\mu}^{(1)}}\left[F\left(\Lambda_{t-s}\left(\delta_{\xi_{s}}\right)\left(\alpha^{s}\right)\right)\right] \\
& =\int_{E} \mathbb{E}_{Q_{x}} F\left(\Lambda_{t-s}\left(\delta_{x}\right)\right) \mu(d x) \\
& =\int_{E}\left(\mathcal{T}_{t-s} F\right)\left(\delta_{x}\right) \mu(d x) \\
& =M_{t-s}^{\mu}(F) .
\end{aligned}
$$


In a similar manner it is seen that

$$
\mathbb{E}_{R_{\mu}^{(1)}}\left[F\left(\bar{\pi}_{s, t}^{(0)}\right)\right]=m_{t-s}^{\mu}(F) .
$$

A straightforward application of martingale convergence theorem shows that as $s \rightarrow-\infty$, almost surely the measure $\bar{\pi}_{s, t}^{(0)}$ converges weakly to the measure $\bar{\pi}_{t}^{(0)}$ defined as follows: For bounded and continuous function $f$ on $E$

$$
\bar{\pi}_{t}^{(0)}(f) \doteq \mathbb{E}_{R_{\mu}^{(1)}}\left[f\left(\xi_{t}\right) \mid \mathcal{Z}_{-\infty}^{t}\right] .
$$

Furthermore we have that (cf. Lemma 3.3 of Kunita [9])

$$
\bar{\pi}_{s, t}^{(1)}(f)=\mathbb{E}_{R_{\mu}^{(1)}}\left[f\left(\xi_{t}\right) \mid \mathcal{Z}_{-\infty}^{t} \vee \mathcal{G}_{-\infty}^{s}\right] .
$$

and thus by the reverse martingale convergence theorem we have that as $s \rightarrow-\infty, \bar{\pi}_{s, t}^{(1)}$ converges weakly to the measure $\bar{\pi}_{t}^{(1)}$ defined as

$$
\bar{\pi}_{t}^{(1)}(f) \doteq \mathbb{E}_{R_{\mu}^{(1)}}\left[f\left(\xi_{t}\right) \mid \mathcal{Z}_{-\infty}^{t} \vee \mathcal{G}_{-\infty}^{-\infty}\right] .
$$

Thus in view of (6.2) and (6.3) we have that $M_{s}^{\mu}$ and $m_{s}^{\mu}$ converge weakly as $s \rightarrow \infty$ to the law of $\bar{\pi}_{t}^{(0)}, \bar{\pi}_{t}^{(1)}$ respectively, which also shows that the laws of $\bar{\pi}_{t}^{(0)} \bar{\pi}_{t}^{(1)}$ are independent of $t$. Denote these laws as $m^{\mu}$ and $M^{\mu}$ respectively. Also note that since $m^{\mu}$ and $M^{\mu}$ are the limits of $m_{t}^{\mu}$ and $M_{t}^{\mu}$ as $t \rightarrow \infty$ and $\left(\mathcal{T}_{t}\right)$ is a Feller semigroup, both $m^{\mu}$ and $M^{\mu}$ have to be $\left(\mathcal{T}_{t}\right)$ invariant. We now recall the following definition from [9].

Definition 6.1 A measure $\nu \in \mathcal{P}(E)$ is the barycenter of the measure $\Phi \in$ $\mathcal{P}(\mathcal{P}(E))$ if and only if for every $\varphi \in C_{b}(E)$,

$$
\nu(\varphi)=\int_{\mathcal{P}(E)} \nu^{\prime}(\varphi) \Phi\left(d \nu^{\prime}\right) .
$$

From Theorem 3.2 of Kunita [9] we have that both $m^{\mu}$ and $M^{\mu}$ have barycenters $\mu$ and if $\Phi$ is another $\mathcal{T}_{t}$ invariant measure with barycenter $\mu$ then for all $F \in C_{c}(E)$,

$$
m^{\mu}(F) \leq \Phi(F) \leq M^{\mu}(F)
$$

Next recalling that $m^{\mu}$ and $M^{\mu}$ are the laws of $\bar{\pi}_{t}^{(0)}$ and $\bar{\pi}_{t}^{(1)}$ respectively, we have that $m^{\mu}$ equals $M^{\mu}$ if $\mathcal{G}_{-\infty}^{-\infty}$ is trivial. From (6.4) we observe that the equality of $m^{\mu}$ and $M^{\mu}$ implies that $\mathcal{T}_{t}$ admits a unique invariant measure with barycenter $\mu$. We thus have the following result. 
Theorem 6.2 Suppose that there is a unique $\left(T_{t}\right)$ invariant measure $\mu$.

Then there is a unique $\mathcal{T}_{t}$ invariant measure if for all $f \in C_{b}(E)$

$$
\limsup _{t \rightarrow \infty} \int_{E}\left|T_{t} f(x)-\mu(f)\right| \mu(d x)=0 .
$$

Proof: As pointed out in [9], (6.5) is equivalent to the condition that $\mathcal{G}_{-\infty}^{-\infty}$ is trivial. This proves the uniqueness of $\left(\mathcal{T}_{t}\right)$ invariant measure.

We remark that our proof of the theorem above is different from the proof in $([9])$. The proof in [9] requires showing that $\left(\bar{\pi}_{t}^{(0)}\right)$ and $\left(\bar{\pi}_{t}^{(1)}\right)$ are Markov with semigroup $\mathcal{T}_{t}$ which is shown in [9] by appealing to the uniqueness of the solution to Kushner-Stratonovich equation. Though we don't need the Markov properties for our proof above, nevertheless using the Feller property of $\mathcal{T}_{t}$, they are seen to hold as argued below.

Proposition 6.3 Let $\mu$ be a $\left(T_{t}\right)$ invariant measure. For $i=0,1,\left(\bar{\pi}_{t}^{(i)}\right)$ is a stationary Markov process on $\left(\Omega^{1}, \mathcal{B}\left(\Omega^{1}\right), R_{\mu}^{(1)}\right)$ with semigroup $\mathcal{T}_{t}$.

Proof: Let $g \in C_{b}(\mathcal{P}(E))$ and fix $-\infty<u<t<\infty$. Then from an application of martingale convergence theorem and the observation that a.s., $\bar{\pi}_{s, t}^{(0)}$ converges weakly to the measure $\bar{\pi}_{t}^{(0)}$ as $s \rightarrow-\infty$ we have that

$$
\begin{aligned}
\mathbb{E}\left(g\left(\bar{\pi}_{t}^{(0)}\right) \mid \mathcal{Z}_{-\infty}^{u}\right) & =\lim _{s \rightarrow-\infty} \mathbb{E}\left(g\left(\bar{\pi}_{s, t}^{(0)}\right) \mid \mathcal{Z}_{s}^{u}\right) \\
& =\lim _{s \rightarrow-\infty} \mathbb{E}\left(g\left(\Lambda_{t-s}(\mu)\left(\alpha^{s}\right)\right) \mid \sigma\left(\alpha_{v}^{s} ; 0 \leq v \leq u-s\right)\right) \\
& =\lim _{s \rightarrow-\infty} g_{1}\left(\Lambda_{u-s}(\mu)\left(\alpha^{s}\right)\right) \\
& =\lim _{s \rightarrow-\infty} g_{1}\left(\bar{\pi}_{s, u}^{(0)}\right) \\
& =g_{1}\left(\bar{\pi}_{u}^{(0)}\right)
\end{aligned}
$$

where all the limits in (6.6) are taken in probability and $g_{1}$ is the real valued function on $\mathcal{P}(E)$ defined in (4.33) with $s$ there replaced by $u$, i.e.,

$$
g_{1}(\nu) \doteq \mathbb{E}_{Q_{\nu}}\left[g\left(\Lambda_{t-u}(\nu)\right)\right] .
$$

Note that $g_{1}$ is independent of $s$ and the last step in the above display follows on observing that the Feller property of $\mathcal{T}_{t}$ implies that $g_{1}$ is continuous. This 
proves the Markov property of $\left(\bar{\pi}_{t}^{(0)}\right)$ and that the semigroup for this Markov process is $\left(\mathcal{T}_{t}\right)$. As is already seen that the law of $\left(\bar{\pi}_{t}^{(0)}\right)$ does not depend on $t$ and thus this Markov process is stationary. Finally consider the process $\left(\bar{\pi}_{t}^{(1)}\right)$. Let $g$ be as before. Then

$$
\begin{aligned}
\mathbb{E}_{R_{\mu}^{(1)}} & {\left[g\left(\bar{\pi}_{t}^{(1)}\right) \mid \mathcal{Z}_{-\infty}^{u} \vee \mathcal{G}_{-\infty}^{-\infty}\right] } \\
& =\lim _{s \rightarrow-\infty} \mathbb{E}_{R_{\mu}^{(1)}}\left[g\left(\bar{\pi}_{s, t}^{(1)}\right) \mid \mathcal{Z}_{-\infty}^{u} \vee \mathcal{G}_{-\infty}^{s}\right] \\
& =\lim _{s \rightarrow-\infty} \mathbb{E}_{R_{\mu}^{(1)}}\left[g\left(\bar{\pi}_{s, t}^{(1)}\right) \mid \mathcal{Z}_{s}^{u} \vee \mathcal{G}_{-\infty}^{s}\right] \\
& =\lim _{s \rightarrow-\infty} \mathbb{E}_{R_{\mu}^{(1)}\left[g\left(\Lambda_{t-s}\left(\delta_{\xi_{s}}\right)\left(\alpha^{s}\right)\right) \mid \sigma\left(\alpha_{v}^{s}: 0 \leq v \leq u-s\right) \vee \mathcal{G}_{-\infty}^{s}\right]} \\
& =\lim _{s \rightarrow-\infty} g_{1}\left(\Lambda_{u-s}\left(\delta_{\xi_{s}}\right)\left(\alpha^{s}\right)\right) \\
& =\lim _{s \rightarrow-\infty} g_{1}\left(\bar{\pi}_{s, u}^{(1)}\right) \\
& =g_{1}\left(\bar{\pi}_{u}^{(1)}\right)
\end{aligned}
$$

where $g_{1}$ is as in (6.7). Hence, $\left(\bar{\pi}_{t}^{(1)}\right)$ is a Markov process with semigroup $\left(\mathcal{I}_{t}\right)$. The stationarity of this process follows as before.

We now turn our attention to the semigroup $\left(\mathcal{S}_{t}\right)$. Let $\mu$, as before, be a $\left(T_{t}\right)$ invariant probability measure. Define the probability measures $\bar{m}_{t}^{\mu}$ and $\bar{M}_{t}^{\mu}$ on $(\mathcal{P}(E) \times E)$ as follows. For $A \in \mathcal{B}(\mathcal{P}(E)) \otimes \mathcal{B}(E)$,

$$
\bar{m}_{t}^{\mu}(A)=\int_{E}\left(\mathcal{S}_{t} \mathcal{I}_{A}\right)(\mu, x) \mu(d x)
$$

and

$$
\bar{M}_{t}^{\mu}(A)=\int_{E}\left(\mathcal{S}_{t} \mathcal{I}_{A}\right)\left(\delta_{x}, x\right) \mu(d x) .
$$

Observe that for real measurable bounded function $F$ on $\mathcal{P}(E) \times E$ and $-\infty<s<t<\infty$

$$
\begin{aligned}
\mathbb{E}_{R_{\mu}^{(1)}}\left[F\left(\bar{\pi}_{s, t}^{(1)}, \xi_{t}\right)\right] & =\mathbb{E}_{R_{\mu}^{(1)}}\left[F\left(\Lambda_{t-s}\left(\delta_{\xi_{s}}\right)\left(\alpha^{s}\right), \xi_{t}\right)\right] \\
& =\int_{E} \mathbb{E}_{\hat{R}_{x}} F\left(\Lambda_{t-s}\left(\delta_{x}\right), \xi_{t-s}\right) \mu(d x) \\
& =\int_{E}\left(\mathcal{S}_{t-s} F\right)\left(\delta_{x}, x\right) \mu(d x) \\
& =\bar{M}_{t-s}^{\mu}(F) .
\end{aligned}
$$

In a similar way it is seen that

$$
\mathbb{E}_{R_{\mu}^{(1)}}\left[F\left(\bar{\pi}_{s, t}^{(0)}, \xi_{t}\right)\right]=\bar{m}_{t-s}^{\mu}(F) .
$$


Now the almost sure convergence, as $s \rightarrow-\infty$, of $\left(\bar{\pi}_{s, t}^{(0)}, \xi_{t}\right)$ and $\left(\bar{\pi}_{s, t}^{(1)}, \xi_{t}\right)$ to $\left(\bar{\pi}_{t}^{(0)}, \xi_{t}\right)$ and $\left(\bar{\pi}_{t}^{(1)}, \xi_{t}\right)$ respectively implies that $\bar{m}_{u}^{\mu}$ and $\bar{M}_{u}^{\mu}$ converge weakly to the law of $\left(\bar{\pi}_{t}^{(0)}, \xi_{t}\right)$ and $\left(\bar{\pi}_{t}^{(1)}, \xi_{t}\right)$ respectively and also that these laws don't depend on $t$. Denote the law of $\left(\bar{\pi}_{t}^{(0)}, \xi_{t}\right)$ by $\bar{m}^{\mu}$ and the law of $\left(\bar{\pi}_{t}^{(1)}, \xi_{t}\right)$ by $\bar{M}^{\mu}$. Once more the Feller property of the semigroup $\left(\mathcal{S}_{t}\right)$ implies that since $\bar{m}_{t}^{\mu}$ and $\bar{M}_{t}^{\mu}$ converge weakly to $\bar{m}^{\mu}$ and $\bar{M}^{\mu}$ respectively, these latter measures are $\left(\mathcal{S}_{t}\right)$ invariant. Next note that for $C \in \mathcal{B}(\mathcal{P}(E))$

$$
\bar{m}^{\mu}(C \times E)=m^{\mu}(C)
$$

and

$$
\bar{M}^{\mu}(C \times E)=M^{\mu}(C) .
$$

Further note that for real bounded measurable functions $f$ and $F$ on $E$ and $\mathcal{P}(E)$ respectively:

$$
\begin{aligned}
\int_{\mathcal{P}(E) \times E} F(\nu) f(x) \bar{m}^{\mu}(d \nu, d x) & =\mathbb{E}_{R_{\mu}^{(1)}}\left[\left(F\left(\bar{\pi}_{t}^{(0)}\right) f\left(\xi_{t}\right)\right)\right] \\
& =\mathbb{E}_{R_{\mu}^{(1)}\left[F\left(\bar{\pi}_{t}^{(0)}\right) \mathbb{E}_{R_{\mu}^{(1)}}\left(f\left(\xi_{t}\right) \mid \mathcal{Z}_{-\infty}^{t}\right)\right]} \\
& =\int_{E} F(\nu) \nu(f) m^{\mu}(d \nu) .
\end{aligned}
$$

Similarly,

$$
\int_{\mathcal{P}(E) \times E} F(\nu) f(x) \bar{M}^{\mu}(d \nu, d x)=\int_{E} F(\nu) \nu(f) M^{\mu}(d \nu) .
$$

Thus $\bar{m}^{\mu}, \bar{M}^{\mu}$ are $\left(\mathcal{S}_{t}\right)$ invariant measures for which (5.5) holds. Also, in the class of $\left(\mathcal{S}_{t}\right)$ invariant probability measures for which $(5.5)$ holds, $\bar{m}^{\mu}$ is the minimal and $\bar{M}^{\mu}$ is the maximal in the sense of Kunita [10], (cf. Theorem $2.2,[10])$. Finally note that if $\mathcal{G}_{-\infty}^{-\infty}$ is trivial, $\left(\bar{\pi}_{t}^{(0)}, \xi_{t}\right)=\left(\bar{\pi}_{t}^{(1)}, \xi_{t}\right)$ a.s. and thus $\bar{m}^{\mu}=\bar{M}^{\mu}$. Hence the following theorem holds.

Theorem 6.4 Suppose that there is a unique $\left(T_{t}\right)$ invariant measure $\mu$ and suppose that (6.5) holds for all $f \in C_{b}(E)$. Then there is a unique $\left(\mathcal{S}_{t}\right)$ invariant measure for which (5.5) holds. 


\section{Asymptotic Stability}

In this section, we will study the asymptotic behavior of the filter when it is initialized at an incorrect initial condition. The results extend the results of Ocone-Pardoux [11] to the case of unbounded $h$ and also we do away with the assumption in [11] that the state space is locally compact.

We will once more use the notations of Section 5 and assume continuity of $h$. In particular recall that the signal process $X$ is an $E$ valued time homogeneous Markov process with a Feller semigroup $T_{t}$ and initial distribution (law of $\left.X_{0}\right) \gamma$. Further, we assume that $T_{t}$ admits a unique invariant probability measure $\mu$ and that (6.5) holds. We have noted in the previous section that in this case, the semigroup $\mathcal{T}_{t}$ is Feller and also admits a unique invariant probability measure. Here is the first result on asymptotic stability

Theorem 7.1 Suppose that $\gamma$ satisfies

$$
\gamma T_{t} \rightarrow \mu
$$

Let

$$
\pi_{t}(B)(\omega)=\Lambda_{t}(\gamma, B)(Y .(\omega))
$$

be the filter. Then the law of $\pi_{t}$ on $(\Omega, \mathcal{F}, P)$ converges to $M$ - the invariant measure for the semigroup $\mathcal{T}_{t}$.

Proof: This result can be proved exactly following the steps in the proof of Theorem 3 in Stettner [12]. Note that we have already proved the Feller property of $\mathcal{T}_{t}$ and hence it follows that if $\nu_{n}$ converges to $\nu$, then $m_{t}^{\nu_{n}}$ converges in law to $m_{t}^{\nu}$. This is a crucial step in the proof of Theorem 3 in [12]. The tightness of $\left\{m_{t}^{\gamma}: t \geq 0\right\}$ follows from (7.1) exactly as in Stettner. Also, we have already proved that there exists a unique $\mathcal{T}_{t^{-}}$ invariant probability measure $M$.

The previous result gives the asymptotics of the filter $\pi_{t}$ when the signal process is a purely non-deterministic ergodic Markov process. 
Ocone-Pardoux [11] consider the behavior of the filter when the initial distribution of the signal is wrongly taken to be $\nu$ instead of $\gamma$. So where as the correct filter is given by (7.2), the incorrectly initialized filter is given as:

$$
\bar{\pi}_{t}(B)(\omega)=\Lambda_{t}(\nu, B)(Y .(\omega) .
$$

In [11] it was shown that under appropriate conditions

$$
\mathbb{E}_{P}\left[\left(\left\langle\pi_{t}, \varphi\right\rangle-\left\langle\bar{\pi}_{t}, \varphi\right\rangle\right)^{2}\right] \rightarrow 0
$$

as $t$ tends to $\infty$. The paper [11] assumes that $h$ is bounded and that $E$ is locally compact. We will now show that (7.4) holds in our framework.

We need the following auxiliary result for this purpose.

Theorem 7.2 Let $\gamma^{n}, \nu^{n} \in \mathcal{P}(E)$ be such that

$$
\gamma^{n} \rightarrow \mu, \quad \nu^{n} \rightarrow \mu
$$

Let $T>0$ be fixed. Then for a bounded continuous function $\varphi$ on $E$,

$$
\mathbb{E}_{Q_{\gamma^{n}}}\left[\left|\left\langle\Lambda_{T}\left(\gamma^{n}\right), \varphi\right\rangle-\left\langle\Lambda_{T}\left(\nu^{n}\right), \varphi\right\rangle\right|\right] \rightarrow 0
$$

Proof: In view of (4.28)-(4.29)

$$
\mathbb{E}_{Q_{\gamma^{n}}}\left[\left|\left\langle\Lambda_{T}\left(\gamma^{n}\right), \varphi\right\rangle-\left\langle\Lambda_{T}\left(\nu^{n}\right), \varphi\right\rangle\right|\right]=\mathbb{E}_{Q}\left[\left|\left\langle\Lambda_{T}\left(\gamma^{n}\right), \varphi\right\rangle-\left\langle\Lambda_{T}\left(\nu^{n}\right), \varphi\right\rangle\right| \Gamma_{T}\left(\gamma^{n}, E\right)\right] .
$$

By Theorem 5.1

$$
\left|\left\langle\Lambda_{T}\left(\gamma^{n}\right), \varphi\right\rangle-\left\langle\Lambda_{T}\left(\nu^{n}\right), \varphi\right\rangle\right| \rightarrow 0 \text { in } Q \text { probability }
$$

and

$$
\Gamma_{T}\left(\gamma^{n}, E\right) \text { is } Q \text { - uniformly integrable. }
$$

The required result follows from these observations.

This is the analog of the result in [11] for our setup. 
Theorem 7.3 Let $\nu \in \mathcal{P}(E)$ be given. Suppose that $\nu, \gamma$ satisfy

$$
\gamma T_{t} \rightarrow \mu \quad \nu T_{t} \rightarrow \mu
$$

Further, suppose that

$$
Q_{\gamma} \text { is absolutely continuous with respect to } Q_{\nu}
$$

Then the filter $\pi_{t}$ (defined by (7.2)) and the erroneous filter $\bar{\pi}_{t}$ wrongly computed with initial measure $\nu$ (defined by (7.3)) satisfy

$$
\mathbb{E}_{P}\left[\left(\left\langle\pi_{t}, \varphi\right\rangle-\left\langle\bar{\pi}_{t}, \varphi\right\rangle\right)^{2}\right] \rightarrow 0
$$

Proof: As in [11], let the finite memory approximations $\pi_{t-\tau, t}, \bar{\pi}_{t-\tau, t}$ of $\pi_{t}$, $\bar{\pi}_{t}$ respectively be defined by

$$
\begin{aligned}
\pi_{t-\tau, t} & =\Lambda_{\tau}\left(\gamma T_{t-\tau}\right) \\
\bar{\pi}_{t-\tau, t} & =\Lambda_{\tau}\left(\nu T_{t-\tau}\right) .
\end{aligned}
$$

Then given $\varepsilon>0$, there exists $\tau_{\varepsilon}$ such that for $\tau>\tau_{\varepsilon}$ we have, for $\varphi \in C(E)$

$$
\mathbb{E}_{P}\left[\left(\left\langle\pi_{t}, \varphi\right\rangle-\left\langle\pi_{t-\tau, t}, \varphi\right\rangle\right)^{2}\right] \rightarrow 0
$$

and

$$
\mathbb{E}_{P}\left[\left(\left\langle\bar{\pi}_{t}, \varphi\right\rangle-\left\langle\bar{\pi}_{t-\tau, t}, \varphi\right\rangle\right)^{2}\right] \rightarrow 0
$$

as $t$ tends to infinity. These statements are proven in [11], Lemma 3.4. The proof given there does not use boundedness of $h$ or the underlying assumption in that paper that $E$ is locally compact and hence carries over the present set up. Since $\varphi$ is bounded, say by $K$, we have

$$
\mathbb{E}_{P}\left[\left(\left\langle\pi_{t-\tau, t}, \varphi\right\rangle-\left\langle\bar{\pi}_{t-\tau, t}, \varphi\right\rangle\right)^{2}\right] \leq 2 K \mathbb{E}_{P}\left[\left|\left\langle\pi_{t-\tau, t}, \varphi\right\rangle-\left\langle\bar{\pi}_{t-\tau, t}, \varphi\right\rangle\right|\right]
$$

and hence to complete the proof remains to show that

$$
\lim _{t \rightarrow \infty} \mathbb{E}_{P}\left[\left|\left\langle\pi_{t-\tau, t}, \varphi\right\rangle-\left\langle\bar{\pi}_{t-\tau, t}, \varphi\right\rangle\right|\right]=0 .
$$

This in turn follows from Theorem 7.2 and the assumption (7.6) upon observing that $\pi_{t-\tau, t}=\Lambda_{\tau}\left(\gamma T_{t-\tau}\right)$ and $\bar{\pi}_{t-\tau, t}=\Lambda_{\tau}\left(\nu T_{t-\tau}\right)$.

Acknowledgement: The authors would like to thank Professor T. G. Kurtz who pointed out that (5.1) is a consequence of the Feller property of $\left(T_{t}\right)$. (5.1) had been included as an assumption in an earlier version. 


\section{References}

[1] A.G. Bhatt, G. Kallianpur, and R.L. Karandikar. Uniqueness and robustness of solution of measure-valued equations of nonlinear filtering. The Annals of Probability, 23:1895-1938, 1995.

[2] A.G. Bhatt, G. Kallianpur, and R.L. Karandikar. Robustness of the nonlinear filter. To appear in Stochastic Processes and their applications, 1999.

[3] A.G. Bhatt and R.L. Karandikar. Path continuity of the nonlinear filter. Preprint, 1999.

[4] A. Budhiraja and H.J. Kushner. Approximation and limit results for nonlinear filters over an infinite time interval. To appear in SIAM Journal of Control and Optimization, Technical Report: Lefschetz Center for Dynamical Systems, 1998.

[5] A. Budhiraja and H.J. Kushner. Approximation and limit results for nonlinear filters over an infinite time interval: Part II, random sampling algorithms. To appear in SIAM Journal of Control and Optimization, Technical Report: Lefschetz Center for Dynamical Systems, 1999.

[6] G. Kallianpur. Stochastic filtering theory. Springer-Verlag, New York, 1980.

[7] G. Kallianpur and R. L. Karandikar. White noise theory of Prediction, Filtering and Smoothing. Gordon and Breach, New York, 1988.

[8] R.L. Karandikar. On pathwise stochastic integration. Stochastic Processes and their applications, 57:11-18, 1995.

[9] H. Kunita. Asymptotic behavior of the nonlinear filtering errors of Markov processes. Journal of Multivariate Analysis, 1:365-393, 1971.

[10] H. Kunita. Ergodic properties of nonlinear filtering processes. In K.C. Alexander and J.C. Watkins, editors, Spatial Stochastic Processes, 1991.

[11] D. Ocone and E. Pardoux. Asymptotic stability of the optimal filter with respect to its initial condition. SIAM Journal of Control and Optimization, 34:226-243, 1996. 
[12] L. Stettner. On invariant measures of filtering processes. In K. Helmes, N. Christopeit, and M. Kohlmann, editors, Stochastic differential systems, Proc. 4th Bad Honnef Conf.,1988, Lecture Notes in Control and Inform Sci., pages 279-292, 1989. 